\title{
DERIVAÇÃO DE UMA EQUAÇÃO PARA O CÁLCULO DO TEOR DE UMIDADE DE SEMENTES DE TRIGO UTILIZANDO-SE O FATOR DE QUALIDADE $\mathbf{Q}^{1}$
}

\author{
Pedro Amorim Berbert ${ }^{2}$, Brian Christopher Stenning ${ }^{3}$, Juarez de Sousa e Silva ${ }^{4}$, \\ Daniel Marçal de Queiroz ${ }^{5}$ \& Evandro de Castro Melo ${ }^{6}$
}

\begin{abstract}
RESUMO
Objetivou-se, com este trabalho, obter um modelo para o cálculo do teor de umidade do trigo, independentemente de sua massa específica aparente, $\rho$, a partir da medição simultânea da permissividade elétrica relativa, $\varepsilon^{\prime}$, do produto, em duas frequiências, utilizando-se um medidor do fator de qualidade Q. Avaliaram-se as possíveis fontes de erro durante a medição, em função das diferenças entre os valores experimental e calculado da capacitância do sensor. Verificou-se, também, o efeito causado pela utilização de grânulos de poliestireno expandido para redução artificial do valor da massa específica aparente dos grãos de trigo. O modelo obtido permitiu estimar-se o teor de umidade do trigo, variedade Estica, no intervalo entre 12 e $18 \%$ b.u., com desvio-padrão e erro máximo de 0,8 e 1,1 pontos percentuais de umidade, respectivamente. Concluiu-se que a metodologia proposta não permite, para as condições estudadas, a obtenção de um modelo para a estimativa do teor de umidade do trigo e que seja independente de $\rho$.
\end{abstract}

Palavras-chave: instrumentação, teor de umidade, trigo

\section{DERIVATION OF A DENSITY-INDEPENDENT EQUATION FOR ESTIMATION OF WHEAT MOISTURE CONTENT USING THE QUALITY FACTOR Q}

\footnotetext{
ABSTRACT

The object of this research work was to develop a density-independent equation to estimate the moisture content of wheat by simultaneously measuring the relative permittivity, $\varepsilon^{\prime}$, of the product at two different frequencies using a Q-Meter. Owing to the difference observed between the measured and theoretical capacitance of the empty sample container, an analysis of errors that arise during the measurement procedure was carried out. The effect due to the presence of polystyrene beads on the dielectric properties of wheat, which were used to artificially reduce the bulk density of the seeds, was also investigated. The resulting equation was capable of estimating wheat moisture content, variety

${ }^{1}$ Apoio financeiro da International Foundation for Science (IFS) da Fundação de Amparo à Pesquisa do Estado de Minas Gerais (FAPEMIG) e da Fundação Coordenação de Aperfeiçoamento de Pessoal de Nível Superior (CAPES)

${ }^{2}$ Doutor, FAPEMIG, Ph.D. em Engenharia Agrícola, Universidade Federal de Viçosa, Departamento de Engenharia Agrícola, CEP 36571 - 000, Viçosa, MG. Fone: (031) 899 1919. E-mail: pberbert@ mail.ufv.br

${ }^{3}$ Professor, B.Sc., CIAgrE., Cranfield University, Silsoe College, Postharvest Technology Department, MK45 4DT, Silsoe, Bedfordshire, UK. E-mail: stbcs@ @ilsoe.cranfield.ac.uk

${ }^{4}$ Professor Titular, Universidade Federal de Viçosa, Departamento de Engenharia Agrícola, Viçosa, MG

${ }^{5}$ Professor Adjunto I, Universidade Federal de Viçosa, Departamento de Engenharia Agrícola, Viçosa, MG

${ }^{6}$ Professor Adjunto II, Universidade Federal de Viçosa, Departamento de Engenharia Agrícola, Viçosa, MG
} 
Estica, in the range from 12 to $18 \%$ w.b. with a standard deviation and a maximum error of 0.8 and 1.1 percentage points moisture, respectively. It was concluded that the proposed method does not allow the derivation of a bulk density-independent model to estimate wheat moisture content for the conditions studied.

Key words: instrumentation, moisture content, wheat

\section{INTRODUÇÃO}

O teor de umidade dos grãos durante a colheita pode variar gradualmente ao longo dos dias ou das semanas, caso o processo dure o suficiente para que ocorram estas mudanças, ou pode alterar-se abruptamente quando se muda de um campo de cultivo para outro. Em situações em que ocorrem variações significativas no teor de umidade inicial dos grãos, é possível demonstrar-se que a adoção de mecanismos de controle automático, na etapa de secagem, é um procedimento capaz de propiciar tanto um produto final mais homogêneo quanto de evitar um consumo excessivo de energia durante o processo (McFarlane, 1987; Eltigani \& Bakker-Arkema, 1987); no entanto, a adoção de sistemas de monitoração automática do processo de secagem, principalmente nos EUA e na Europa, tem ocorrido de forma mais gradual do que as pesquisas faziam supor. As razões desta ocorrência foram discutidas amplamente por diversos pesquisadores (Eltigani \& Bakker-Arkema, 1987; Nybrant, 1989; Moreira \& Bakker-Arkema, 1990) mas os problemas essenciais se resumem aos seguintes fatores:

a) no caso dos sistemas de monitoração do teor de umidade baseados na temperatura do ar de exaustão, a causa principal da ocorrência de falhas reside no fato de não haver uma relação bem definida entre o teor de umidade final do produto, na saída do secador, e a temperatura do ar de exaustão (Brooker et al., 1992);

b) nos casos em que são empregados medidores elétricos de teor de umidade, a principal desvantagem advém do fato de eles serem capazes de avaliar apenas amostras localizadas e, portanto, não representativas da massa de grãos;

c) nos sistemas onde se mede diretamente a variável controlada, ou seja, o teor de umidade do produto, a maior dificuldade, tanto nos medidores que utilizam a capacitância quanto naqueles que utilizam freqüências de microondas, devese ao efeito da massa específica aparente dos grãos. Conforme demonstrado por Kraszewski \& Kulinski (1976) um grau maior ou menor de compactação dos grãos, no compartimento eletricamente ativo do sensor, terá grande influência no resultado final indicado pelo medidor.

Além do controle automático de secadores, a recepção e amostragem de grãos em unidades de pré-processamento de produtos agrícolas é uma outra atividade que poderia se beneficiar de um medidor que permitisse a avaliação contínua e em linha do teor de umidade dos produtos. Atualmente, devido à retirada de amostras localizadas, o manejo de unidades armazenadoras baseia-se em dados pouco confiáveis, uma vez que a avaliação dos riscos de deterioração e perda técnica é feita utilizando-se valores médios do teor de umidade dessas amostras. A mistura de lotes de grãos secos e úmidos visando à obtenção de um produto com teor de umidade médio considerado adequado para uma armazenagem segura, é uma prática comum tanto em fazendas como em grandes unidades de pré-processamento de grãos. Christensen \& Kaufmann (1969) relataram experimentos conduzidos por diversos pesquisadores em que ocorreram perdas significativas do produto armazenado devido à presença de grãos com elevado teor de umidade na mistura de diversos lotes. Atualmente, utilizando-se métodos de amostragem convencionais, é praticamente impossível detectar a presença de grãos úmidos por meio dos medidores de teor de umidade existentes no mercado.

Com o desenvolvimento de um medidor em linha, a avaliação contínua do teor de umidade do produto a ser recebido permitiria um manejo mais adequado de uma unidade de préprocessamento de grãos e cereais, reduzindo os riscos envolvidos e permitindo melhor avaliação do percentual de perdas durante o armazenamento. Neste caso, a variação da massa específica aparente de grãos em movimento continua a ser fator limitante ao desenvolvimento de medidores capazes de avaliar, continuamente e em linha, o teor de umidade na recepção dos produtos em unidades de secagem e armazenamento.

Várias metodologias têm sido propostas para reduzir ou eliminar a influência da massa específica aparente na determinação em linha do teor de umidade de produtos agrícolas, cujas soluções encontradas se baseiam, geralmente, na determinação de equações empíricas envolvendo parâmetros dielétricos do produto que sejam independentes da massa específica, uma vez que seria muito difícil, senão impossível, controlar este parâmetro em uma coluna de grãos em movimento.

Nos últimos 25 anos, a maioria dos pesquisadores que buscavam soluções para o efeito da massa específica aparente na determinação em linha do teor de umidade de produtos agrícolas, concentrou seus esforços no desenvolvimento de medidores operando na freqüência de microondas (Okabe et al., 1973; Kraszewski \& Kulinski, 1976; Meyer \& Schilz, 1980; Kress-Rogers \& Kent, 1987). Argumentava-se que esses medidores teriam, em teoria, maior potencial para reduzir o efeito negativo da condutividade iônica da amostra devido à eliminação do contato físico entre os grãos e as células eletricamente ativas do medidor, o que não ocorreria, ou ocorreria em menor grau, nos medidores operando em radiofrequiências. A redução da condutividade iônica propiciaria a obtenção de equações empíricas mais precisas. Esperava-se, também, que os custos envolvidos no desenvolvimento de produtos utilizando circuitos de microondas sofressem reduções além das que realmente ocorreram nos últimos anos.

No entanto, é relevante observar que, na última década, as pesquisas visando ao desenvolvimento de medidores em linha estão voltadas para a utilização de capacitores operando em frequiências de rádio (Eichler, 1985; Channa, 1985; Shaw, 1989; Lawrence \& Nelson, 1993; Lawrence et al., 1994). Isto se deve, entre outros fatores, ao fato de que, com a escolha apropriada do tamanho e forma dos eletrodos, seria possível medir-se o teor de umidade utilizando-se um volume considerável de grãos, resultando em uma leitura mais representativa. Acrescente-se a 
isto o fato de os custos para o desenvolvimento e a construção de aparelhos que utilizam circuitos operando em baixa frequiência, serem consideravelmente mais reduzidos que aqueles operando em freqüências de microondas, tornando-os mais atraentes para o eventual utilizador.

McFarlane (1987) propôs um método para eliminar a influência da variação da massa específica aparente no grau de exatidão da determinação do teor de umidade de produtos agrícolas granulares, o qual se baseia na solução simultânea de duas equações empíricas que relacionam a permissividade elétrica relativa, $\varepsilon^{\prime}$, do produto ao seu teor de umidade e à sua massa específica aparente, em duas freqüências de oscilação do campo elétrico. Sendo assim, com o presente trabalho objetivou-se derivar uma equação para o cálculo do teor de umidade de sementes de trigo, utilizando-se a metodologia proposta originalmente por McFarlane (1987) e empregando-se um medidor do fator de qualidade $\mathrm{Q}$ de um circuito elétrico para medir as propriedades dielétricas do produto.

\section{MATERIAL E MÉTODOS}

\section{Descrição do circuito de medição}

$\mathrm{O}$ instrumento utilizado neste trabalho para determinação das propriedades dielétricas do trigo foi o medidor do fator de qualidade Q, modelo TF 1245, fabricado por Marconi Instruments Ltd., St. Albans, UK. Este instrumento permite a medição da capacitância e do fator de qualidade $\mathrm{Q}$ dos componentes de um circuito e, conseqüentemente, a determinação indireta da permissividade elétrica relativa, $\varepsilon$ ', e da tangente do ângulo de perdas, $\operatorname{tg} \delta$, de amostras de materiais dielétricos. Apresenta-se, na Figura 1, o diagrama do circuito do instrumento contendo seus componentes mais relevantes.

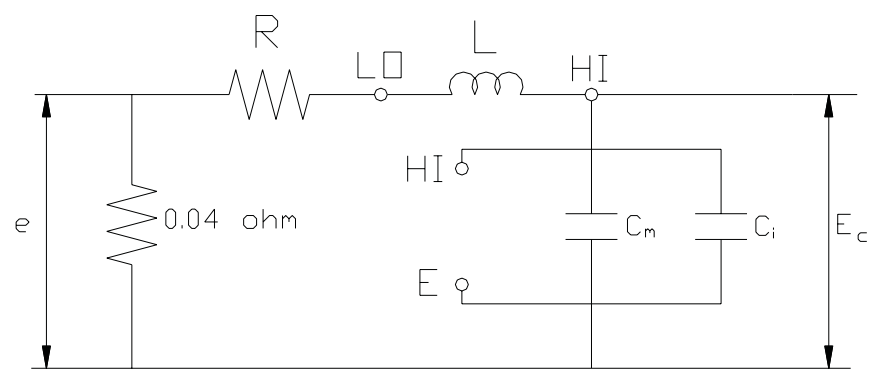

Figura 1. Componentes básicos do circuito do medidor do fator $\mathrm{Q}$

O instrumento é composto por um oscilador capaz de induzir sinais no intervalo entre $1 \mathrm{kHz}$ e $300 \mathrm{MHz}$ e produzir uma diferença de potencial $e=22 \mathrm{~V}_{\text {ef }}$ em um circuito ressonante RLC série. A porção capacitiva do medidor é composta por um capacitor principal, $\mathrm{C}_{\mathrm{m}}$, que pode ser ajustado para qualquer valor de capacitância entre 20 e 500 pF e por um capacitor de ajuste fino, $C_{i}$, que permite pequenas alterações $( \pm 5 \mathrm{pF})$ na capacitância do circuito. O ramal capacitivo possui, também, um par de terminais externos (HI e E) para que o elemento sensor possa ser conectado em paralelo com $\mathrm{C}_{\mathrm{m}}$ e $\mathrm{C}_{\mathrm{i}}$. Além desses componentes, o instrumento possui, ainda, um par adicional de terminais (LO-HI) para que um indutor, L, possa ser inserido, em série, com a combinação em paralelo de $\mathrm{C}_{\mathrm{m}}$ e $\mathrm{C}_{\mathrm{i}}$. Os indutores disponíveis para utilização variavam entre $0,2 \mu \mathrm{H}$ e $25 \mathrm{mH}$. O instrumento é também equipado com um voltímetro ligado em paralelo à porção capacitiva $\left(\mathrm{E}_{\mathrm{C}}\right)$. Se o valor de " $e$ " for mantido constante, selecionando-se um valor apropriado de freqüência e indutância, o circuito apresentado na Figura 1 poderá ser submetido a ressonância ajustando-se o valor de $\mathrm{C}_{\mathrm{m}}$. Neste caso, o fator de qualidade do circuito é dado por $\mathrm{Q}=\left(\mathrm{E}_{\mathrm{C}} / e\right)$.

\section{Descrição do elemento sensor}

O dispositivo escolhido para conter as amostras durante as medições das propriedades dielétricas foi um capacitor de cilindros concêntricos (Fig. 2). Os dois eletrodos do capacitor foram construídos de tubos de latão com 155,0 mm de comprimento. O cilindro externo tinha diâmetro interno de 126,5 mm e parede com espessura de $3,0 \mathrm{~mm}$ e o interno diâmetro externo de 79,6 mm e parede com espessura de 1,5 mm. Para se manter os dois cilindros em posição concêntrica, utilizou-se uma base anular de 15,5 mm de espessura construída de polihexametileno, termoplástico conhecido comercialmente como nylon 66.

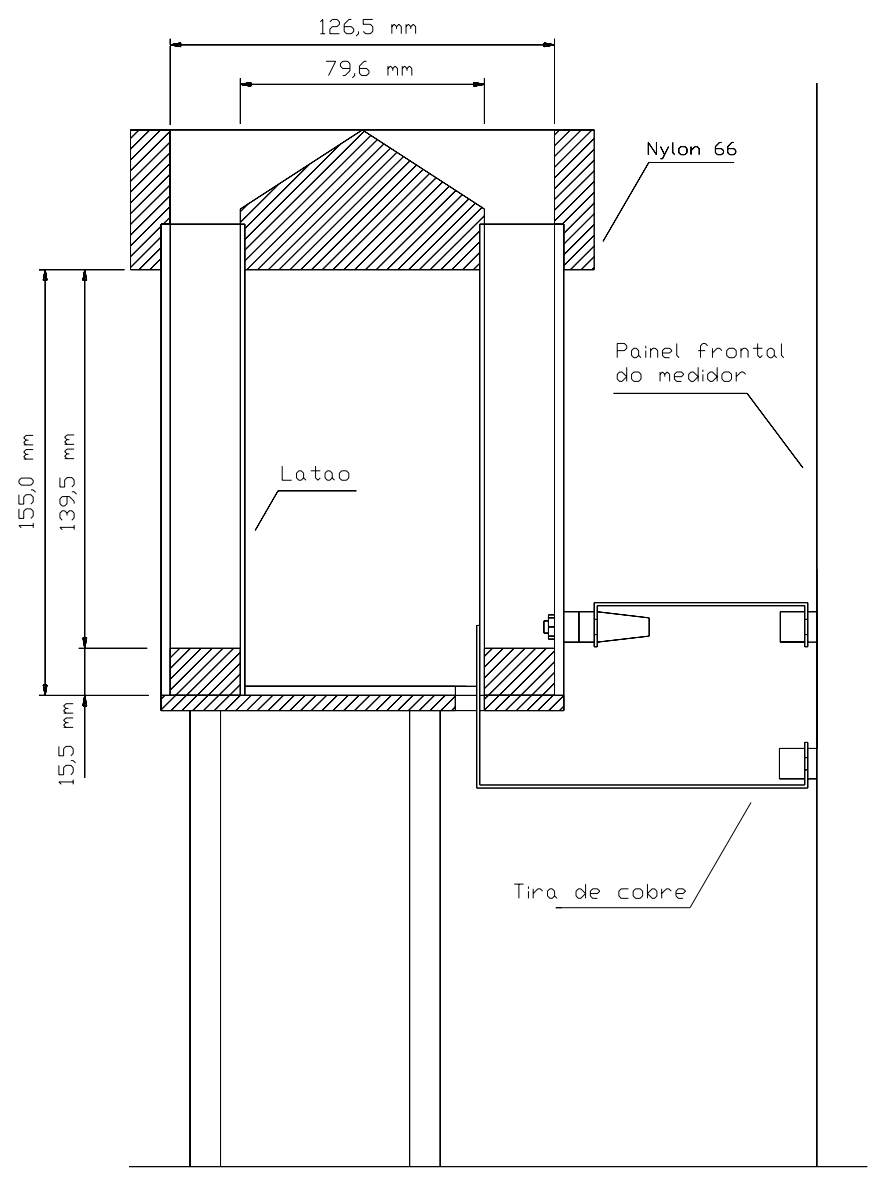

Figura 2. Elemento sensor conectado ao painel frontal do medidor do fator $\mathrm{Q}$

Para facilitar o preenchimento do capacitor com as amostras de trigo, fixou-se um anel de proteção sobre o eletrodo externo e um cone sobre o eletrodo interno, ambos construídos com nylon 66. O volume livre do capacitor era de $1,3806 \times 10^{-3} \mathrm{~m}^{3}$, capaz de conter aproximadamente $1,1 \mathrm{~kg}$ de trigo com teor de umidade de $13,3 \%$ b.u. A metodologia para o cálculo das propriedades dielétricas de amostras de sementes de trigo, utilizando-se o circuito do medidor do fator Q (Fig. 1) e o elemento sensor (Fig. 2) encontra-se descrita em Berbert et al. (1999). 


\section{Preparação das amostras de trigo}

Todas as medições de propriedades dielétricas foram realizadas utilizando-se sementes certificadas de trigo (Triticum aestivum L.) variedade Estica. O lote inicial do produto encontrava-se com teor de umidade de $14,2 \%$ b.u. e, depois de ser subdividido em amostras de $1,5 \mathrm{~kg}$, foi armazenado em frascos herméticos de vidro sob temperatura de aproximadamente $8^{\circ} \mathrm{C}$, em câmaras climáticas. Para a obtenção de amostras com teores de umidade entre 14,2 e 22,0\% b.u., com incrementos de 2 pontos percentuais de umidade, adicionavam-se quantidades predeterminadas de água destilada às amostras iniciais. Nelson (1977) afirma que os medidores elétricos que se baseiam na medição da capacitância deixam de apresentar resultados confiáveis para teores de umidade superiores a $22 \%$ b.u., sendo esta a razão para que se limitasse o maior valor de teor de umidade a $22 \%$ b.u.

Para a obtenção de amostras com teores de umidade de $12 \mathrm{e}$ $10,5 \%$ b.u., as amostras com teor de umidade inicial de $14,2 \%$ b.u. foram secas a temperatura de aproximadamente $21^{\circ} \mathrm{C} \mathrm{em} \mathrm{um}$ protótipo de secador de leito fixo. Depois dos tratamentos de reumedecimento e secagem, as amostras foram armazenadas a $5^{\circ} \mathrm{C}$ por, no mínimo, 15 dias e, durante o período de armazenamento, eram revolvidas três vezes ao dia para evitar a formação de gradientes de umidade entre os grãos e permitir maior homogeneidade na distribuição de umidade. As alterações no teor de umidade das amostras durante o experimento nunca ultrapassaram 0,2 ponto percentual. $\mathrm{O}$ teor de umidade dos grãos foi determinado de acordo com as Regras Britânicas de Testes para Cereais e Leguminosas (British Standard Methods of Test for Cereals and Pulses, 1992).

\section{Massa específica}

Adotou-se o seguinte procedimento para se determinar o efeito da massa específica aparente no cálculo do teor de umidade da amostra, a partir de suas propriedades dielétricas. Para se obter valores naturais de massa específica aparente vertia-se, inicialmente, a amostra em um funil de plástico posicionado acima do capacitor; logo a seguir, acionava-se uma válvula com dispositivo de abertura rápida, localizada na extremidade inferior do funil, distante $30 \mathrm{~mm}$ do capacitor, permitindo que a amostra fluísse naturalmente (Fig. 3). O material em excesso era retirado do capacitor com uma régua, mantendo-se os grãos sempre no mesmo nível das extremidades superiores dos eletrodos.

Para se reduzir o valor da massa específica aparente, misturavam-se as amostras de trigo com uma quantidade predeterminada de grânulos de poliestireno expandido com diâmetro de aproximadamente $3 \mathrm{~mm}$. A constante dielétrica do poliestireno, no intervalo entre $1 \mathrm{kHz}$ e $1 \mathrm{GHz}$, possui o valor de 1,017, muito semelhante, em termos práticos, à constante dielétrica do ar, $\varepsilon^{\prime}$ ar $=1,0006$ (Griffin \& Skochdopole, 1964; Kraus \& Carver, 1973). O poliestireno é também um material que provoca uma dissipação de energia extremamente reduzida da onda eletromagnética, apresentando fator de perda dielétrica $\varepsilon^{\prime \prime}=0,0001$.

Para aumentar em 5\% a massa específica aparente da amostra, separava-se uma quantidade adicional de grãos que correspondia a um aumento de $5 \%$ na massa relativa à massa específica natural. O capacitor era preenchido com a amostra, como descrito anteriormente, e depois colocado para vibrar, por $3 \mathrm{~min}$, a $40 \mathrm{~Hz}$, com amplitude de $0,1 \mathrm{~mm}$; a seguir, a quantidade adicional de grãos era despejada no capacitor, que era novamente vibrado por um período adicional de 3 min. A massa específica aparente, em todos os casos, foi calculada dividindo-se a massa da amostra pelo volume livre do capacitor. Foram realizadas três repetições para cada combinação de teor de umidade e massa específica aparente.

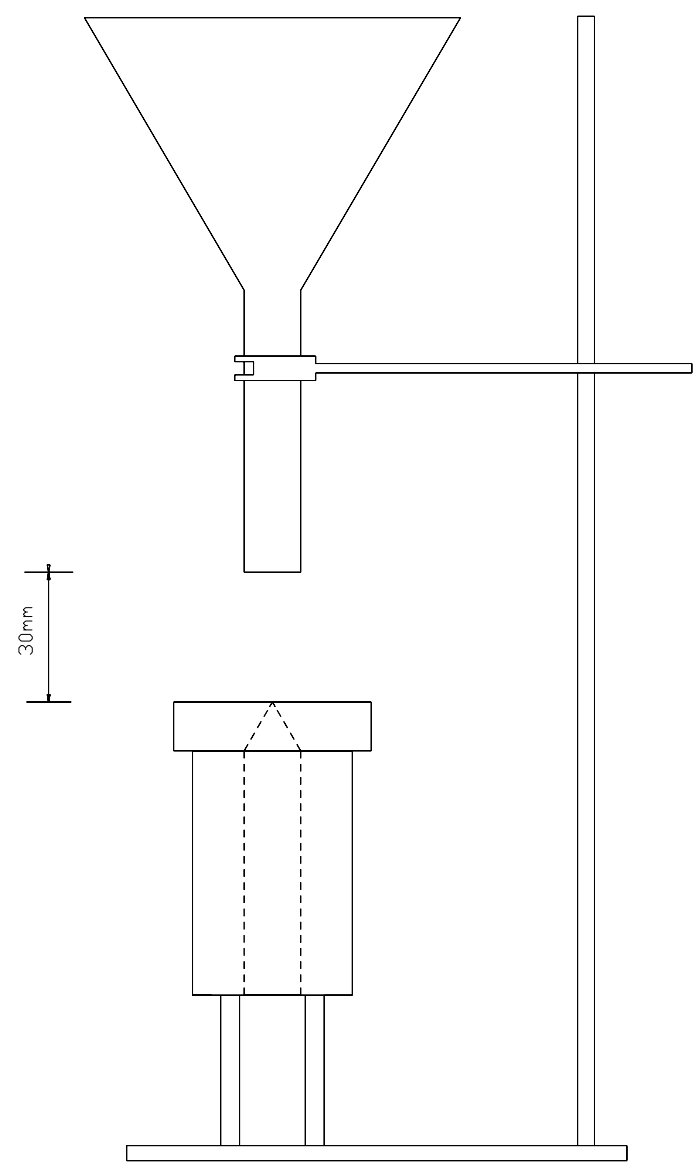

Figura 3. Dispositivo para obtenção da massa específica natural do produto

Desenvolvimento de um modelo para o cálculo do teor de umidade do trigo, independentemente de sua massa específica aparente, utilizando-se a medição simultânea de $\varepsilon$ ' em duas freqüências de oscilação

McFarlane (1987) ao modificar uma metodologia originalmente proposta por Dzhemella \& Pustynnikov (1965) propôs um novo método para reduzir a influência da variação da massa específica aparente na acurácia da determinação do teor de umidade de produtos granulares. Este método baseia-se na solução simultânea de duas equações empíricas que relacionam a permissividade elétrica relativa, $\varepsilon^{\prime}$, do produto ao seu teor de umidade (U, \% b.u.) e à sua massa específica aparente $\left(\rho, \mathrm{kg} \mathrm{m}^{-3}\right)$ em duas frequiências de oscilação do campo elétrico. Apresentam-se, a seguir, as equações obtidas por McFarlane (1987), a 0,1 e $10 \mathrm{MHz}$, para amostras de trigo com teores de umidade de $14,7,16,7$ e $19,5 \%$ b.u. e massa específica variando no intervalo entre 688 a $794 \mathrm{~kg} \mathrm{~m}^{-3}$.

$$
\begin{gathered}
\varepsilon_{(0,1 \mathrm{MHz})}^{\prime}=0,559 \mathrm{U}+0,00698 \rho-9,27 \\
\varepsilon_{(10 \mathrm{MHz})}^{\prime}=0,144 \mathrm{U}+0,00506 \rho-2,29
\end{gathered}
$$


Manipulando-se as Eqs. 1 e 2 de forma a eliminar o parâmetro $\rho$ e obter uma única equação que correlacionasse o teor de umidade aos parâmetros dielétricos das amostras de trigo, obteve-se o seguinte modelo:

$$
\mathrm{U}=2,78 \varepsilon_{0,1 \mathrm{MHz}}^{\prime}-3,83 \varepsilon_{10 \mathrm{MHz}}^{\prime}+17,00
$$

McFarlane (1987) verificou que era possível se estimar o teor de umidade do trigo, utilizando-se a Eq. 3, com desviopadrão e erro máximo de 0,6 e 1,0 ponto percentual de umidade, respectivamente, em relação aos valores determinados em estufa.

\section{RESULTADOS E DISCUSSÃO}

\section{Análise dos erros envolvidos no processo de medição}

Antes da medição das propriedades dielétricas do trigo, mediu-se a capacitância do capacitor vazio no intervalo de freqüência selecionado, cujos resultados são apresentados na Tabela 1. Observa-se que os valores variaram entre 28 e $30 \mathrm{pF}$, havendo-se obtido uma média de 29,32 pF; calculando-se, então, a capacitância teórica do elemento sensor, foi possível determinar-se o valor aproximado das capacitâncias espúrias presentes no circuito de medição.

Admitindo-se o valor de 4,2 para a permissividade relativa do nylon 66 a $1 \mathrm{MHz}$ (Kinney, 1961) considerando-se que $\varepsilon^{\prime}{ }_{\text {ar }}=1,0006$ (Kraus \& Carver, 1973) e se utilizando a seguinte equação para a capacitância por unidade de comprimento de um capacitor coaxial ideal, com a =0,0398 m e b $=0,0633$, obtém-se o valor de 24,57 pF para a capacitância teórica do capacitor.

em que:

$$
\frac{\mathrm{C}}{\ell}=\frac{2 \pi \varepsilon_{0} \varepsilon_{\mathrm{d}}^{\prime}}{\ln \left(\frac{\mathrm{b}}{\mathrm{a}}\right)}
$$

C - capacitância, $\mathrm{pF}$

$\ell$ - comprimento, $\mathrm{m}$

$\varepsilon_{0}$ - permissividade do vácuo, $8,85 \mathrm{pF} \mathrm{m}^{-1}$

$\varepsilon_{\mathrm{d}}^{\prime}$ - permissividade relativa do dielétrico localizado entre os condutores, adimensional

b - raio interno do condutor externo, $\mathrm{m}$

a - raio do condutor interno, $\mathrm{m}$.
Para se obter um valor mais exato, é necessário adicionar-se a capacitância em virtude da presença do anel de proteção (nylon 66) localizado sobre o eletrodo externo do capacitor. A capacitância introduzida no circuito devido à introdução deste anel, foi medida no intervalo de 0,1 a $10 \mathrm{MHz}$, utilizando-se um medidor LCR modelo 4285 A, fabricado pela Hewlett Packard, tendo variado de 0,38 a $0,47 \mathrm{pF}$, com uma média de $0,43 \mathrm{pF}$. Desta forma, a capacitância teórica do elemento sensor é de $25,0 \mathrm{pF}$.

$\mathrm{O}$ grau de precisão das medições feitas com o medidor do fator de qualidade $\mathrm{Q}$ empregado neste trabalho, foi determinado medindo-se a capacitância de diversos capacitores ordinários com valores nominais da mesma ordem de grandeza do elemento sensor; os valores medidos sempre estiveram no intervalo entre $\pm 0,5 \mathrm{pF}$ em relação aos valores nominais. Considerando-se que os valores de capacitância dos capacitores encontrados no mercado podem variar consideravelmente, mesmo entre aqueles provenientes de um mesmo lote de fabricação, o grau de precisão foi considerado aceitável para atender aos objetivos deste trabalho. Sendo assim, a diferença entre os valores medido e calculado da capacitância do elemento sensor, 4,32 pF, não se deve a problemas relacionados à falta de precisão do instrumento de medição. Para explicar esta diferença, é preciso se entender que, além das capacitâncias espúrias, a Eq. 4 não está totalmente de acordo com a realidade, pois não considera a distorção das linhas de fluxo do campo elétrico nas extremidades dos eletrodos do capacitor. Apresenta-se, a seguir, uma discussão sobre as possíveis causas para o surgimento da discrepância entre os valores medido e calculado.

Matthews (1963) afirmou que, na prática, a capacitância de um capacitor vazio seria sempre maior que sua capacitância teórica (Eq. 4) devido ao efeito dos materiais utilizados na construção do sensor; no entanto, não se encontraram evidências na literatura que possam corroborar esta afirmação.

Não se espera que a auto-capacitância do indutor utilizado no circuito de medição seja fator que possa limitar o grau de exatidão do valor medido, uma vez que ela é parte integrante da capacitância total do capacitor principal do instrumento de medição, tanto com o elemento sensor conectado ou não ao circuito; desta forma, sua influência é eliminada quando se subtrai, do valor da capacitância medida com o elemento sensor

Tabela 1. Capacitância do capacitor vazio, para diversos valores de frequiência e indutância, obtida utilizando-se o medidor do fator de qualidade Q

\begin{tabular}{cccccc}
\hline $\begin{array}{c}\text { Freqüência } \\
(\mathrm{MHz})\end{array}$ & $\begin{array}{c}\text { Indutância } \\
(\mu \mathrm{H})\end{array}$ & $\begin{array}{c}\text { Capacitância do capacitor vazio } \\
(\mathrm{pF})\end{array}$ & $\begin{array}{c}\text { Freqüência } \\
(\mathrm{MHz})\end{array}$ & $\begin{array}{c}\text { Indutância } \\
(\mu \mathrm{H})\end{array}$ & $\begin{array}{c}\text { Capacitância do capacitor vazio } \\
(\mathrm{pF})\end{array}$ \\
\hline 0,1 & 10000 & 30 & 2,0 & 25,0 & 28 \\
0,2 & 2500 & 29 & 3,0 & 10,0 & 30 \\
0,3 & 1000 & 30 & 4,0 & 5,0 & 30 \\
0,4 & 500 & 29 & 5,0 & 2,5 & 29 \\
0,5 & 500 & 30 & 6,0 & 1,5 & 29 \\
0,6 & 200 & 29 & 7,0 & 1,5 & 30 \\
0,7 & 200 & 29 & 8,0 & 1,0 & 30 \\
0,8 & 100 & 29 & 9,0 & 1,0 & 29 \\
0,9 & 100 & 29 & 10,0 & 1,0 & \\
1,0 & 75 & & & & \\
\hline
\end{tabular}


conectado, o valor da capacitância sem que qualquer elemento esteja conectado ao circuito (Berbert et al., 1999), mas a autocapacitância em paralelo com a indutância L, interfere na acurácia dos valores medidos de $\mathrm{Q}$, porque a capacitância ressonante efetiva deixa de ser devida exclusivamente à capacitância permanente do circuito. Conseqüentemente, a diferença de potencial elétrico que surge na seção capacitiva do circuito é menor do que realmente seria, caso a auto-capacitância do indutor não estivesse presente. Este fato tem implicações diretas na confiabilidade dos valores do fator de perda dielétrica ( $\varepsilon ")$ estimados a partir do fator de qualidade Q (Marconi Instruments, 1990).

Parece razoável, então, considerar-se que parte da diferença entre os valores medido e calculado se deve ao efeito de todos os componentes resistivos e reativos que existem entre o elemento sensor e os terminais do medidor do fator Q; sendo assim, um valor medido incluirá tanto as constantes do próprio elemento sensor quanto aquelas devidas às tiras de cobre utilizadas para sua conexão ao equipamento de medição (Fig. 1). Para que esta questão fique mais clara, o par de tiras de cobre pode ser considerado capacitor de placas planas paralelas conectado eletricamente em paralelo com o sensor; além disso, existe ainda a combinação de uma auto-indutância e uma resistência em paralelo com o capacitor formado pelas tiras de cobre. Para finalizar esta questão, pode-se considerar que a combinação de todos estes parâmetros elétricos seja equivalente a um único valor de capacitância $\mathrm{C}$ e resistência $\mathrm{R}$, em paralelo, valores efetivamente reconhecidos pelo medidor do fator Q. A capacitância efetiva do capacitor de placas planas paralelas formado pelas tiras de cobre, foi calculada utilizando-se a seguinte equação, $\mathrm{C}=\left[\left(\varepsilon^{\prime}{ }_{\text {ar }} \varepsilon_{0} A\right) / d\right]$ em que $A$ é a área de uma das tiras $\left(9,8 \times 10^{-4} \mathrm{~m}^{2}\right), d$ é a distância que as separa $\left(5,4 \times 10^{-2} \mathrm{~m}\right)$ e $\varepsilon_{0}$ representa a permissividade do vácuo $\left(8,85 \mathrm{pF} \mathrm{m}^{-1}\right)$ [Kraus $\&$ Carver, 1973]. O valor encontrado foi de $0,16 \mathrm{pF}$.

A auto indutância deste componente diminui o efeito da reatância capacitiva do circuito pelo fato de estarem em fases opostas, significando que a capacitância medida do circuito será maior que o valor real, porém as tiras de cobre foram construídas segundo as instruções do fabricante do medidor do fator Q de forma a minimizar, tanto quanto possível, os erros induzidos pela presença de condutores adicionais; desta forma, não se espera que a auto-indutância do par de tiras possa explicar por completo a diferença entre os valores medido e calculado.

Nelson (1979) analisou a influência da auto-indutância do elemento sensor no valor medido de sua capacitância quando se emprega um medidor do fator Q. Para tanto, utilizou dois circuitos em paralelo, representando um dielétrico com elevado fator de perda ( $\varepsilon ")$, com e sem a presença de uma indutância residual no ramal capacitivo dos circuitos (Figuras 4A e 4B). Para determinado valor de freqüência, um capacitor pode ser representado pela combinação, em paralelo, de sua capacitância ideal, C', e uma resistência pura, R. As propriedades dielétricas de uma amostra de grãos inserida entre os eletrodos do capacitor, a esta freqüiência, têm como base estes valores aparentes, C' e $\mathrm{R}$, efetivamente medidos.

Supondo-se que a indutância residual, L, exista em série com a capacitância, C, o circuito mostrado na Figura 4B é mais representativo da realidade que aquele mostrado na Figura $4 \mathrm{~A}$. A impedância do ramal reativo deste circuito será $Z_{L C}=j\left(X_{L}-X_{C}\right)$.
Caso o circuito seja interpretado como capacitância pura (Fig. 4A) sua impedância será $\mathrm{Z}_{\mathrm{C}^{\prime}}=-\mathrm{j} \mathrm{X}_{\mathrm{C}}$. Igualando-se essas impedâncias, obtém-se uma equação para o cálculo do valor de C', ou seja,

$$
\begin{gathered}
-j X_{C^{\prime}}=j\left(X_{L}-X_{C}\right) \therefore X_{C^{\prime}}=X_{L}-X_{C} \\
-\frac{1}{\omega C^{\prime}}=\omega L-\frac{1}{\omega C} \\
C^{\prime}=\frac{C}{1-\omega^{2} L C}
\end{gathered}
$$
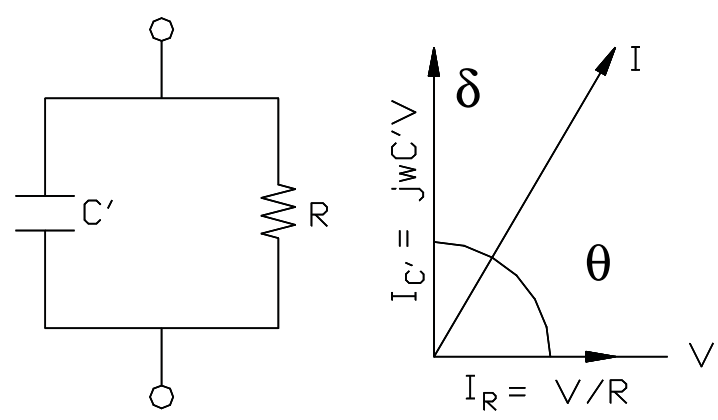

(A)
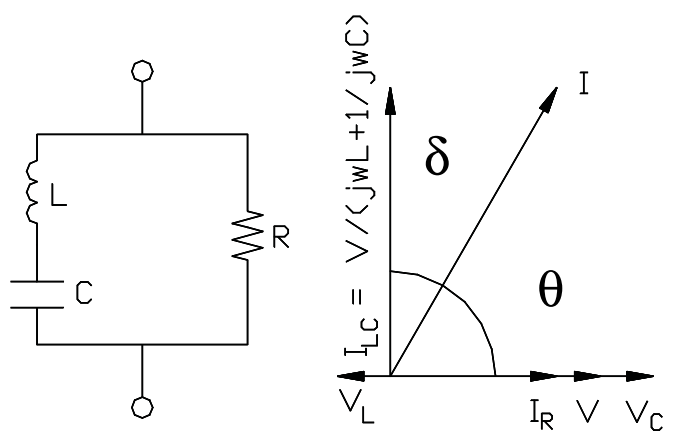

(B)

Figura 4. Representação de um circuito elétrico contendo um dielétrico com elevado fator de perda, $\varepsilon$ ", $\operatorname{sem}(\mathrm{A})$ e com (B) uma indutância residual em série no seu ramal capacitivo Nelson (1979)

Conseqüentemente, se L é diferente de zero, a capacitância medida é um valor aparente e será maior que o valor verdadeiro. Embora a análise anterior seja uma simplificação das condições reais, a discussão ajuda a ilustrar as possíveis conseqüências de ignorar-se a presença de indutâncias residuais, que podem acarretar problemas quando se utiliza o medidor do fator de qualidade Q, para se medir valores de capacitância. Nelson (1979) sugere que, para freqüências de até $100 \mathrm{MHz}$, o valor medido pode ser considerado uma representação adequada do valor real e que não há necessidade de correções; além disso, o fabricante do medidor do fator $\mathrm{Q}$ afirma que os problemas relacionados à presença de indutâncias residuais só se tornam significativos para valores elevados de capacitância $(\mathrm{C} \geq 100 \mathrm{pF})$ medidos em freqüências superiores a $50 \mathrm{MHz}$ (Marconi Instruments, 1990). 
A diferença ainda existente entre os valores medido e calculado pode ser explicada pela presença de diversos outros componentes resistivos e reativos que não foram abordados. Por exemplo, há que se considerar a capacitância dos conectores banhados a níquel e utilizados para unir o elemento sensor ao instrumento de medição e a capacitância que se origina devido ao contato de materiais diferentes; além disso, consideram-se, ainda, os erros que se originam devido à geometria imperfeita do elemento sensor, ou seja, os eletrodos não constituem capacitor ideal de cilindros concêntricos. Considerando-se que a medição dos valores de capacitância foi realizada pelo método das diferenças, com o circuito sob ressonância, é razoável considerar-se que os erros que se originam durante o processo de medição seriam da mesma ordem de grandeza, tanto para o capacitor vazio quanto com o preenchido com o material dielétrico (Berbert et al., 1999). Desta forma, os efeitos dos erros seriam eliminados, podendo-se esperar, então, medidas acuradas de suas propriedades dielétricas.

\section{Capacitância do elemento sensor preenchido com uma mistura heterogênea de grânulos de poliestireno e ar}

Apresentam-se, na Tabela 2, os valores das leituras feitas no mostrador do capacitor principal do medidor do fator $\mathrm{Q}$, tanto para o capacitor vazio $\left(\mathrm{C}_{2}\right)$ quanto para o capacitor preenchido com grânulos de poliestireno $\left(\mathrm{C}_{3}\right)$. Mostram-se, também, os valores da permissividade elétrica relativa, $\varepsilon^{\prime}$, desses grânulos, calculados em todo o intervalo de frequiência estudado, de acordo

Tabela 2. Capacitância do elemento sensor vazio $\left(\mathrm{C}_{2}\right)$ e do elemento sensor preenchido com uma mistura de grânulos de poliestireno expandido e ar $\left(\mathrm{C}_{3}\right)$, obtida por meio de um medidor do fator de qualidade $\mathrm{Q}$, e os valores resultantes da permissividade elétrica relativa da mistura

\begin{tabular}{|c|c|c|c|}
\hline \multirow{2}{*}{$\begin{array}{c}\text { Freqüência } \\
\text { MHz }\end{array}$} & \multicolumn{2}{|c|}{$\begin{array}{l}\text { Leitura de capacitância no } \\
\text { mostrador principal do } \\
\text { medidor do fator Q }\end{array}$} & \multirow[t]{2}{*}{$\begin{array}{l}\text { Permissividade } \\
\text { elétrica relativa }\end{array}$} \\
\hline & $\begin{array}{c}\mathrm{C}_{2} \\
(\mathrm{pF})\end{array}$ & $\begin{array}{c}\mathrm{C}_{3} \\
(\mathrm{pF})\end{array}$ & \\
\hline 0,1 & 216 & 216 & 1,00 \\
\hline 0,2 & 215 & 215 & 1,00 \\
\hline 0,3 & 245 & 245 & 1,00 \\
\hline 0,4 & 286 & 285 & 1,05 \\
\hline 0,5 & 170 & 170 & 1,00 \\
\hline 0,6 & 322 & 321 & 1,05 \\
\hline 0,7 & 226 & 225 & 1,05 \\
\hline 0,8 & 354 & 353 & 1,05 \\
\hline 0,9 & 273 & 272 & 1,05 \\
\hline 1,0 & 293 & 293 & 1,00 \\
\hline 2,0 & 228 & 228 & 1,00 \\
\hline 3,0 & 234 & 234 & 1,00 \\
\hline 4,0 & 274 & 274 & 1,00 \\
\hline 5,0 & 376 & 376 & 1,00 \\
\hline 6,0 & 440 & 440 & 1,00 \\
\hline 7,0 & 314 & 314 & 1,00 \\
\hline 8,0 & 346 & 345 & 1,05 \\
\hline 9,0 & 265 & 264 & 1,05 \\
\hline 10,0 & 207 & 207 & 1,00 \\
\hline
\end{tabular}

com a metodologia apresentada em Berbert et al. (1999). Os valores de $\varepsilon^{\prime}$ obtidos experimentalmente variaram no intervalo $1,00 \leq \varepsilon^{\prime} \leq 1,05$, havendo sido considerados representações razoáveis do valor esperado, $\varepsilon^{\prime}=1,01$, calculado de acordo com a seguinte equação, proposta por Looyenga (1965) para a permissividade elétrica relativa de uma mistura heterogênea de dois dielétricos. $O$ valor obtido, $\varepsilon^{\prime}=1,01$, foi calculado utilizando-se $\mathrm{v}_{2}=0,8, \varepsilon^{\prime}{ }_{1}=1,0006$ e $\varepsilon_{2}^{\prime}=1,017$.

$$
\varepsilon^{\prime}=\left\{\left[\left(\varepsilon_{2}^{\prime}\right)^{1 / 3}-\left(\varepsilon_{1}^{\prime}\right)^{1 / 3}\right] \mathrm{v}_{2}+\left(\varepsilon_{1}^{\prime}\right)^{1 / 3}\right\}^{3}
$$

em que:

$\mathrm{v}_{2}$ - fração, em volume, do componente disperso

$\varepsilon_{2}^{\prime}$ - permissividade elétrica relativa do componente disperso $\varepsilon_{1}{ }_{1}$ - permissividade elétrica do outro componente

\section{Permissividade elétrica relativa}

Apresenta-se, na Figura 5 e 6, a variação da permissividade elétrica relativa de sementes de trigo, variedade Estica, com

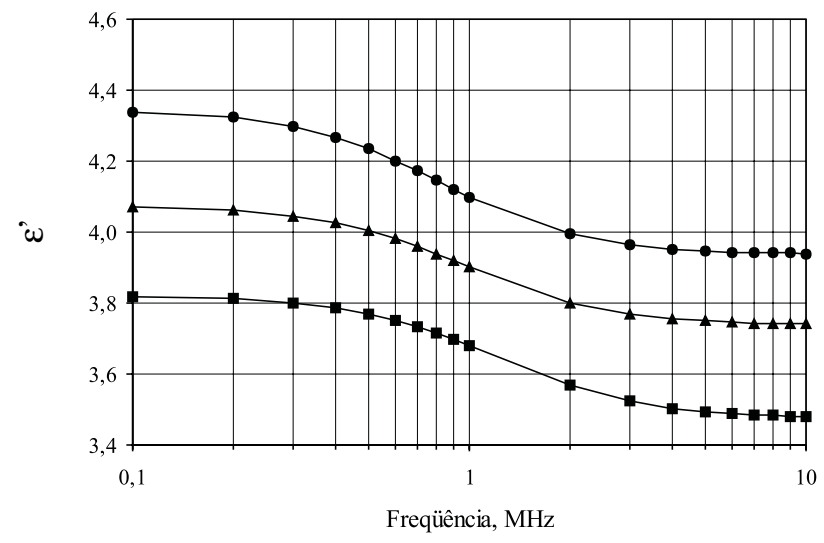

Figura 5. Variação da permissividade elétrica relativa, $\varepsilon^{\prime}$, de sementes de trigo, variedade Estica, em função da freqüência, para teor de umidade de $12 \%$ b.u. e valores indicados de massa específica aparente. $\mathbf{\square}, 717 \mathrm{~kg} \mathrm{~m}^{-3} ; \mathrm{s}, 797 \mathrm{~kg} \mathrm{~m}^{-3} ; \bullet$, $826 \mathrm{~kg} \mathrm{~m}^{-3}$

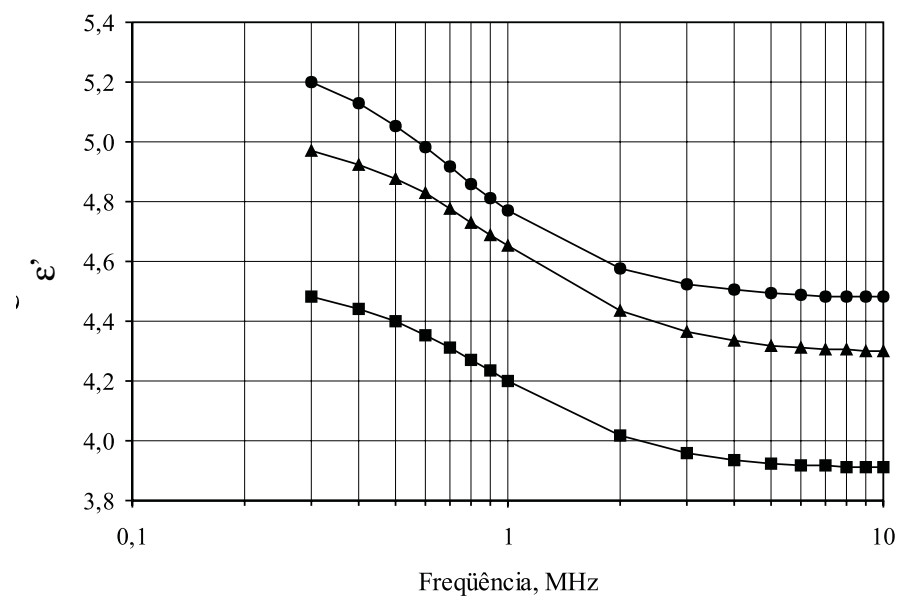

Figura 6. Variação da permissividade elétrica relativa, $\varepsilon^{\prime}$, de sementes de trigo, variedade Estica, em função da frequiência, para teor de umidade de $16 \%$ b.u. e valores indicados de massa específica aparente. $\mathrm{n}, 699 \mathrm{~kg} \mathrm{~m}^{-3} ; \mathrm{s}, 760 \mathrm{~kg} \mathrm{~m}^{-3} ; 1,795 \mathrm{~kg} \mathrm{~m}^{-3}$ 
teores de umidade de 12 e $16 \%$ b.u., respectivamente, em função da freqüência, para diferentes valores de massa específica aparente. Explicações para o formato das curvas e a magnitude dos valores obtidos podem ser encontradas em Berbert \& Stenning (1997).

\section{Modelo para o cálculo do teor de umidade}

Utilizou-se a análise de regressão múltipla para correlacionar os valores medidos de $\varepsilon^{\prime}$ ao teor de umidade (U, \% b.u.) e massa específica aparente $\left(\rho, \mathrm{kg} \mathrm{m}^{-3}\right)$ das amostras de trigo. Obtiveram-se funções do tipo $\mathrm{U}=\mathrm{f}\left(\varepsilon^{\prime}, \rho\right)$ e $\rho=\mathrm{f}\left(\varepsilon^{\prime}, \mathrm{U}\right)$ para 17 valores de frequiência entre $300 \mathrm{kHz}$ e $10 \mathrm{MHz}$. Apresentam-se, na Tabela 3, as equações obtidas por meio de regressão e os respectivos coeficientes de determinação. As equações obtidas a 9 e $10 \mathrm{MHz}$ foram omitidas da Tabela 3 por serem idênticas àquela obtida a 8

Tabela 3. Equações que relacionam a permissividade elétrica relativa, $\varepsilon^{\prime}$, o teor de umidade, $(12,1 \% \leq \mathrm{U} \leq 18,0 \%)$ e a massa específica aparente, $\rho\left(\mathrm{kg} \mathrm{m}^{-3}\right)$, de sementes de trigo, variedade Estica, para valores indicados de freqüência e temperaturas entre $19 \mathrm{e} 22^{\circ} \mathrm{C}$

\begin{tabular}{|c|c|c|}
\hline $\begin{array}{c}\text { Frequiência } \\
\text { MHz }\end{array}$ & Equação & $r^{2}$ \\
\hline \multirow[t]{2}{*}{0,3} & $U=3,440 \varepsilon^{\prime}-0,0184 \rho+12,805$ & 0,98 \\
\hline & $\rho=171,48 \varepsilon^{\prime}-49,99 U+701,36$ & 0,92 \\
\hline \multirow[t]{2}{*}{0,4} & $\mathrm{U}=3,624 \varepsilon^{\prime}-0,0187 \rho+12,353$ & 0,98 \\
\hline & $\rho=177,63 \varepsilon^{\prime}-49,13 U+670,14$ & 0,92 \\
\hline \multirow[t]{2}{*}{0,5} & $\mathrm{U}=3,831 \varepsilon^{\prime}-0,0191 \rho+11,898$ & 0,98 \\
\hline & $\rho=185,34 \varepsilon^{\prime}-48,47 U+635,61$ & 0,92 \\
\hline \multirow[t]{2}{*}{0,6} & $\mathrm{U}=4,044 \varepsilon^{\prime}-0,0198 \rho+11,702$ & 0,98 \\
\hline & $\rho=188,99 \varepsilon^{\prime}-46,77 U+604,43$ & 0,93 \\
\hline \multirow[t]{2}{*}{0,7} & $\mathrm{U}=4,232 \varepsilon^{\prime}-0,0205 \rho+11,611$ & 0,98 \\
\hline & $\rho=191,30 \varepsilon^{\prime}-45,20 U+580,10$ & 0,93 \\
\hline \multirow[t]{2}{*}{0,8} & $U=4,458 \varepsilon^{\prime}-0,0213 \rho+11,387$ & 0,98 \\
\hline & $\rho=195,44 \varepsilon^{\prime}-43,78 U+549,35$ & 0,93 \\
\hline \multirow[t]{2}{*}{0,9} & $\mathrm{U}=4,634 \varepsilon^{\prime}-0,0223 \rho+11,582$ & 0,98 \\
\hline & $\rho=195,00 \varepsilon^{\prime}-41,96 U+531,86$ & 0,94 \\
\hline \multirow[t]{2}{*}{1,0} & $\mathrm{U}=4,789 \varepsilon^{\prime}-0,0231 \rho+11,648$ & 0,98 \\
\hline & $\rho=196,18 \varepsilon^{\prime}-40,82 U+516,05$ & 0,94 \\
\hline \multirow[t]{2}{*}{2,0} & $U=5,648 \varepsilon^{\prime}-0,0285 \rho+12,957$ & 0,98 \\
\hline & $\rho=192,00 \varepsilon^{\prime}-33,71 U+460,02$ & 0,96 \\
\hline \multirow[t]{2}{*}{3,0} & $U=5,889 \varepsilon^{\prime}-0,0306 \rho+13,834$ & 0,98 \\
\hline & $\rho=187,52 \varepsilon^{\prime}-31,53 U+455,54$ & 0,97 \\
\hline \multirow[t]{2}{*}{4,0} & $U=5,974 \varepsilon^{\prime}-0,0315 \rho+14,266$ & 0,98 \\
\hline & $\rho=185,11 \varepsilon^{\prime}-30,67 U+455,59$ & 0,97 \\
\hline \multirow[t]{2}{*}{5,0} & $U=6,006 \varepsilon^{\prime}-0,0318 \rho+14,426$ & 0,98 \\
\hline & $\rho=184,15 \varepsilon^{\prime}-30,32 U+456,11$ & 0,97 \\
\hline \multirow[t]{2}{*}{6,0} & $\mathrm{U}=6,023 \varepsilon^{\prime}-0,0321 \rho+14,628$ & 0,98 \\
\hline & $\rho=182,73 \varepsilon^{\prime}-29,98 U+457,76$ & 0,97 \\
\hline \multirow[t]{2}{*}{7,0} & $\mathrm{U}=5,966 \varepsilon^{\prime}-0,0320 \rho+14,780$ & 0,98 \\
\hline & $\rho=181,64 \varepsilon^{\prime}-30,09 U+464,13$ & 0,97 \\
\hline \multirow[t]{2}{*}{8,0} & $\mathrm{U}=6,039 \varepsilon^{\prime}-0,0324 \rho+14,815$ & 0,98 \\
\hline & $\rho=181,67 \varepsilon^{\prime}-29,71 U+459,28$ & 0,97 \\
\hline
\end{tabular}

$\mathrm{MHz}$, fato este esperado porque as curvas de $\varepsilon^{\prime}$ em função da frequiência, para todos os níveis de teor de umidade e massa específica estudados, tornavam-se assíntotas para valores de freqüência superiores a $8 \mathrm{MHz}$.

Observa-se, na Tabela 3 que, a medida em que se aumenta a freqüência de oscilação, aumenta-se também o valor do coeficiente de determinação das funções do tipo $\rho=f\left(\varepsilon^{\prime}, U\right)$. Além disso, considerando-se que determinada alteração no teor de umidade resulta em alterações mais pronunciadas no valor de $\varepsilon$ ' no intervalo de baixas freqüências, escolheu-se a equação da massa específica aparente a $8 \mathrm{MHz}$ e as equações do teor de umidade no intervalo entre 0,3 e $0,7 \mathrm{MHz}$, para integrarem o modelo para o cálculo do teor de umidade do trigo, utilizando-se a medição simultânea de $\varepsilon^{\prime}$ em duas frequiências. Apresentam-se, na Tabela 4, as equações para o cálculo do teor de umidade do trigo, independentemente de sua massa específica aparente, obtidas ao se introduzir a equação $\rho=181,67 \varepsilon_{8 \mathrm{MHz}}-29,71 \mathrm{U}+459,28$ nas funções do tipo $\mathrm{U}=\mathrm{f}\left(\varepsilon^{\prime}, \rho\right)$ para os valores selecionados de freqüências.

Tabela 4. Modelos para o cálculo do teor de umidade $(12,1 \% \leq \mathrm{U} \leq 18,0 \%)$ do trigo, variedade Estica, a partir de valores de $\varepsilon$ ' medidos simultaneamente em duas freqüências, para temperaturas entre 19 e $22{ }^{\circ} \mathrm{C}$

\begin{tabular}{cccc}
\hline $\begin{array}{c}\text { Freqüiências } \\
\text { MHz }\end{array}$ & Modelo & \multicolumn{2}{c}{ Erros } \\
\hline 0,3 e 8,0 & $\mathrm{U}=7,59 \varepsilon_{0,3}^{\prime}-7,38 \varepsilon_{8,0}^{\prime}+9,61$ & 0,8 & 1,1 \\
0,4 e 8,0 & $\mathrm{U}=8,16 \varepsilon_{0,4}^{\prime}-7,65 \varepsilon_{8,0}^{\prime}+8,47$ & 0,8 & $-1,0$ \\
0,5 e 8,0 & $\mathrm{U}=8,84 \varepsilon_{0,5}^{\prime}-8,01 \varepsilon_{8,0}^{\prime}+7,21$ & 0,8 & $-1,3$ \\
0,6 e 8,0 & $\mathrm{U}=9,81 \varepsilon_{0,6}^{\prime}-8,73 \varepsilon_{8,0}^{\prime}+6,33$ & 0,9 & $-1,3$ \\
0,7 e 8,0 & $\mathrm{U}=10,82 \varepsilon_{0,7}^{\prime}-9,52 \varepsilon_{8,0}^{\prime}+5,61$ & 0,9 & $-1,3$ \\
\hline
\end{tabular}

As equações apresentadas na Tabela 4 mostram desempenho muito semelhante na estimativa do teor de umidade do trigo, utilizando-se os valores da permissividade elétrica relativa medidos em duas freqüências de oscilação. Observa-se, na mesma tabela, que os desvios obtidos não diferem de forma

Tabela 5. Valores experimentais e estimados da permissividade elétrica relativa, $\varepsilon^{\prime}$, de sementes de trigo, variedade Estica, e valores experimentais e estimados pela Equação 7 do seu teor de umidade

\begin{tabular}{|c|c|c|c|c|c|}
\hline \multirow{2}{*}{$\begin{array}{c}\text { Massa } \\
\text { Específica } \\
\text { Aparente } \\
\left(\mathrm{kg} \mathrm{m}^{-3}\right)\end{array}$} & \multicolumn{2}{|c|}{$\begin{array}{l}\text { Permissividade } \\
\text { Elétrica Relativa }\end{array}$} & \multicolumn{3}{|c|}{$\begin{array}{l}\text { Teor de umidade } \\
\text { (\% b.u.) }\end{array}$} \\
\hline & $0,3 \mathrm{MHz}$ & $8,0 \mathrm{MHz}$ & Experimental & Estimado & Erro relativo (\%) \\
\hline 716,8 & 3,80 & 3,48 & 12,1 & 12,8 & $-5,8$ \\
\hline 796,5 & 4,04 & 3,74 & 12,1 & 12,7 & $-5,0$ \\
\hline 826,4 & 4,30 & 3,94 & 12,1 & 13,2 & $-9,1$ \\
\hline 712,0 & 4,15 & 3,76 & 14,2 & 13,4 & $+5,6$ \\
\hline 787,0 & 4,53 & 4,15 & 14,2 & 13,4 & $+5,6$ \\
\hline 821,4 & 4,77 & 4,31 & 14,2 & 14,0 & $+1,4$ \\
\hline 698,7 & 4,48 & 3,91 & 15,7 & 14,8 & $+5,7$ \\
\hline 759,9 & 4,97 & 4,30 & 16,1 & 15,6 & $+3,1$ \\
\hline 795,0 & 5,20 & 4,48 & 15,8 & 16,0 & $-1,3$ \\
\hline 657,6 & 5,03 & 3,95 & 17,9 & 18,6 & $-3,9$ \\
\hline 730,7 & 5,50 & 4,43 & 18,0 & 18,7 & $-3,9$ \\
\hline
\end{tabular}


considerável para os diferentes pares de freqüências; no entanto, como se espera que os valores de $\varepsilon$ ' sejam mais exatos quando medidos em baixos valores de freqüência escolheu-se, entre as equações apresentadas na Tabela 4, a equação seguinte, para estimar o teor de umidade de sementes de trigo, independentemente de sua massa específica aparente.

$$
\mathrm{U}=7,594 \varepsilon_{0,3 \mathrm{MHz}}^{\prime}-7,380 \varepsilon_{8,0 \mathrm{MHz}}^{\prime}+9,611
$$

O desempenho da Eq. 7 foi avaliado ao se comparar os valores estimados de $\mathrm{U}$ com aqueles determinados utilizando-se o método padrão de estufa. Apresentam-se, na Tabela 5, os valores experimentais e estimados para os intervalos de teor de umidade e massa específica aparente avaliados neste trabalho. Observase que a Eq. 7 superestima o teor de umidade para 12 e $18 \%$ b.u. e o subestima para 14,2 e 16,0\% b.u.; além disso, foi possível verificar-se que esta equação é capaz de estimar os valores reais de teor de umidade com desvio-padrão e erro máximo de 0,8 e 1,1 pontos percentuais de umidade, respectivamente. A magnitude desses erros indica que o método da solução simultânea de duas equações empíricas proposto por McFarlane (1987) utilizando-se o medidor do fator de qualidade Q empregado neste trabalho, não é capaz de resolver, com o grau de exatidão esperado, o problema da obtenção de uma equação para a estimativa do teor de umidade de sementes de trigo e que seja totalmente independente da massa específica do produto.

\section{CONCLUSÕES}

1. A magnitude dos erros que surgem durante a medição da capacitância do elemento sensor, utilizando-se o medidor do fator de qualidade Q empregado neste trabalho, não interfere no grau de exatidão e precisão dos valores estimados da permissividade elétrica relativa do trigo.

2. A utilização de grânulos de poliestireno para a redução artificial da massa específica aparente dos grãos tem efeito praticamente desprezível nos valores da permissividade elétrica relativa das sementes de trigo.

3. O método de McFarlane, para as condições estudadas neste trabalho, não permite a obtenção de um modelo para o cálculo do teor de umidade de sementes de trigo que seja independente da massa específica aparente do produto.

\section{REFERÊNCIAS BIBLIOGRÁFICAS}

BERBERT, P.A.; STENNING, B.C. Influência do teor de umidade nas propriedades dielétricas de sementes de trigo no intervalo de $500 \mathrm{kHz}$ a $5 \mathrm{MHz}$. Engenharia Agrícola, Jaboticabal, v.16, n.4, p.47-58, 1997.

BERBERT, P.A.; STENNING, B.C.; QUEIROZ, D.M.; SILVA, J.S. Utilização do fator de qualidade $Q$ na determinação das propriedades dielétricas do trigo. Engenharia Agrícola, Jaboticabal, 1999 (no prelo).

BRITISH STANDARD METHODS OF TEST FOR CEREALS AND PULSES. BS 4317: Part 3: 1993. Determination of moisture content of cereals and cereal products (routine method).

BROOKER, D.B.; BAKKER-ARKEMA, F.W.; HALL, C.W. Drying and storage of grains and oilseeds. New York: van Nostrand Reinhold, 1992. 450p.
CHANNA, K.S. On-line measurement of moisture content of grain in a combine harvester. Silsoe: Cranfield Institute of Technology, 1985. 95p. M.Phil. Thesis.

CHRISTENSEN, C.M.; KAUFMANN, H.H. Grain storage: the role of fungi in quality loss. Minneapolis: University of Minnesota Press, 1969. 139p.

DZHEMELLA, V.V.; PUSTYNNIKOV, V.G. Two-parameter instrument for automatic measurement of moisture content in corn. Instrument Construction, v.7, p.32-4, 1965.

EICHLER, I. On line measurement of grain moisture content on combine harvesters. Silsoe: Cranfield Institute of Technology, 1985.86p. M.Sc. Thesis.

ELTIGANI, A.Y.; BAKKER-ARKEMA, F.W. Automatic control of commercial crossflow grain dryers. Drying Technology, New York, v.5, n.4, p.561-75, 1987.

GRIFFIN, J.D.; SKOCHDOPOLE, R.E. Plastic foams. In: BAER, E. ed. Engineering design for plastics. New York: Reinhold Publishing Corporation, 1964. p.995-1071. Polymer Science and Engineering Series

KINNEY, G.F. Engineering properties and applications of plastics. 2 ed. New York: John Wiley \& Sons Inc., 1961.278p.

KRASZEWSKI, A.; KULINSKI, S. An improved microwave method of moisture content measurement and control. IEEE Transactions on Industrial Electronics and Control Instrumentation, IECI, v.23, n.4, p.364-70, 1976.

KRAUS, J.D.; CARVER, K.R. Electromagnetics. Tokyo: McGraw-Hill Kogakusha Ltd, 1973. 828p.

KRESS-ROGERS, E.; KENT, M. Microwave measurement of powder moisture and density. Journal of Food Engineering, Barking, v.6, p.345-76, 1987.

LAWRENCE, K.C.; NELSON, S.O. Radio-frequency densityindependent moisture determination in wheat. Transactions of the ASAE, St. Joseph, v.36, n.2, p.477-83, 1993.

LAWRENCE, K.C.; WINDHAM, W.R.; NELSON, S.O. Wheat moisture determination by 1 - to $110-\mathrm{MHz}$ swept-frequency admittance measurements. In: ASAE INTERNATIONAL SUMMER MEETING. Kansas City: ASAE Paper № 946051, 1994.

LOOYENGA, H. Dielectric constants of heterogeneous mixtures. Physica, v.31, p.401-6, 1965.

MARCONI INSTRUMENTS LTD. Operating and maintenance handbook. Circuit Magnification Meter TF 1245. St. Albans: Marconi Instruments Ltd., 1990. 44p.

MATTHEWS, J. The design of an electrical capacitance-type moisture meter for agricultural use. Journal of Agricultural Engineering Research, Silsoe, v.8, n.1, p.17-30, 1963.

McFARLANE, N.J.B. Two-frequency capacitance measurement of the moisture content of grain. Silsoe: AFRC Institute of Engineering Research, Divisional Note DN 1434, 1987. 12p.

MEYER, W.; SCHILZ, W. A microwave method for density independent determination of the moisture content of solids. Journal of Physics D: Applied Physics, The Institute of Physics, Great Britain, v.13, p.1823-30, 1980.

MOREIRA, R.G.; BAKKER-ARKEMA, F.W. A feedforward/ feedback adaptive controller for commercial cross-flow grain driers. Journal of Agricultural Engineering Research, Silsoe, v.45, p. 107-16, 1990.

NELSON, S.O. Use of electrical properties for grain-moisture measurement. Journal of Microwave Power, Edmonton, v.12, n.1,p.67-72, 1977.

NELSON, S.O. Improved sample holder for Q-Meter dielectric measurements. Transactions of the ASAE, St. Joseph, v.22, n.4, p.950-54, 1979.

NYBRANT, T.G. Modelling and adaptive control of concurrentflow driers. Computers and Electronics in Agriculture, Amsterdam, v.3, p.243-53, 1989.

OKABE, T.; HUANG, M.T.; OKAMURA, S. A new method for the measurement of grain moisture content by the use of microwaves. Journal of Agricultural Engineering Research, Silsoe, v.18, p.59-66, 1973.

SHAW, S.M.B. The development of a commercial system for the on-line measurement of moisture content in high temperature continuous cereal driers. Silsoe: Cranfield Institute of Technology, 1989. 77p. M.Sc. Thesis. 